\title{
Biochemical and clinical impact of reduced methylene tetrahydrofolate reductase enzyme activity
}

\author{
Samapika Bhaumik ${ }^{1, *}$, Suprava Patel $^{2}$ \\ ${ }^{\mathbf{1}}$ Final Year MBBS Student, ${ }^{2}$ Associate Professor, Dept. of Biochemistry, All India Institute of Medical Sciences, Raipur, Chhattisgarh, \\ India
}

*Corresponding Author:

Email: samapikabhaumik@gmail.com

\begin{abstract}
Methylene tetrahydrofolate reductase (MTHFR) enzyme is a crucial enzyme involved in the pathway of tetrahydrofolate inter-conversion and one-carbon metabolism. Though there are 24 reported polymorphisms associated to MTHFR gene, the two most investigated are C677T (Ala $\left.{ }^{222} \mathrm{Val}\right)$ and A1298C ( $\left.\mathrm{Glu}^{429} \mathrm{Ala}\right)$ single nucleotide polymorphisms (SNPs). The metabolic impact in the form of hyperhomocysteinemia, redistribution of different forms of folates, altered methylation process and accumulation of unmetabolised folic acid have been reported in individuals with reduced or deficient MTHFR activity. These biochemical alterations have been associated with coronary artery diseases, various thromboembolic phenomenon, infertility and reproductive failures, cancers and inflammatory diseases. Folic acid supplementation may further worsen the case in these individuals by increasing the unmetabolised forms. Supplementation of 5MTHF could be a better alternative for therapeutic and preventive purposes.
\end{abstract}

Keywords: MTHFR, Structure, C677T, A1298C, Biochemical impact.

\section{Introduction}

Methylene tetrahydrofolate reductase (MTHFR) enzyme is a crucial enzyme involved in the pathway of tetrahydrofolate inter-conversion and one-carbon metabolism. The prime role of the enzyme is catalyzing the conversion of 5,10 -methylenetetrahydrofolate to 5methyltetrahydrofolate, the major circulatory form of folate that acts as a co-substrate for homocysteine remethylation to methionine by methionine synthase. This homodimer flavoprotein is about $75 \mathrm{kDa}$ consisting of 656 amino acids. Each monomer of the enzyme has 4 important domains: 1) N-terminal catalytic domain, 2) C-terminal regulatory domain, 3) Tryptic cleavage site and 4) Conserved domain. Each N-terminal catalytic domain consists of two noncovalently bound flavin adenine dinucleotide (FAD) as prosthetic group and binds to nicotinamide adenine dinucleotide phosphate (NADPH) as reducing agent. The Cterminus of each monomer has an S-adenosylmethionine binding site which regulates the enzyme activity by allosteric inhibition. The catalytic and regulating domains can be separated by trypsin digestion at the tryptic cleavage site. The enzyme also has a phosphorylation site and phosphorylation reduces enzyme activity by nearly $20 \% .^{1,2}$

This cytosolic enzyme is involved in many biological processes of cellular amino acid metabolism, folic acid metabolic process, homocysteine-methionine cycle, one carbon metabolism, tetrahydrobiopterin and nitric oxide metabolism, blood circulation, neural tube closure, histone methylation and heterochromatin maintenance and various other metabolic activities. ${ }^{3}$

MTHFR Polymorphism: MTHFR is encoded by the MTHFR gene on chromosome 1 location p36.3 in humans and acts as a rate limiting enzyme. Human MTHFR gene is composed of 11 exons and does not have a TATA box but has $\mathrm{CpG}$ islands and multiple potential SP1 binding sites. ${ }^{4,5}$
Though there are 24 reported polymorphisms associated to MTHFR gene, the two most investigated are C677T (rs1801133) and A1298C (rs1801131) single nucleotide polymorphisms (SNPs). ${ }^{6}$

C677T SNP: This is also coined as $\mathrm{Ala}^{222} \mathrm{Val}$. $\mathrm{C}$ at position 677 (leading to an alanine at amino acid 222) is the normal allele. The $677 \mathrm{~T}$ allele (leading to a valine substitution at amino acid 222) encodes a thermolabile enzyme with reduced activity.

Persons with two copies of 677C (677CC) have the most common genotype. 677TT individuals (homozygous) have lower MTHFR activity than CC or CT (heterozygous) individuals. About $10 \%$ of the North American population are T-homozygous for this polymorphism. Ethnic variability in the frequency of the $\mathrm{T}$ allele can be seen viz. frequency is greater in Mediterranean or Hispanics than in Caucasians and Africans or African-Americans. ${ }^{7}$

In persons with 677TT, the degree of enzyme thermolability is more than persons with $677 \mathrm{CT}$ and 677CC. ${ }^{8}$ Owing to less active MTHFR available to produce 5-methyletetrahydrofolate, the persons with 677TT are predisposed to mild hyper-homocysteinaemia. This can also be as a result of low dietary intake of folic acid. However, low folate intake affects individuals with 677TT to a greater extent than those $677 \mathrm{CC}$ or $677 \mathrm{CT}$. Hence, persons with 677TT with lower plasma folate levels are at high risk for elevated plasma homocysteine levels. ${ }^{4}$ 677TT persons are at an increased risk for acute lymphoblastic leukemia ${ }^{9}$ and colon cancer. $^{10}$ Non-Caucasian persons with 677CT genotype are at increased risk of recurrent pregnancy loss. ${ }^{11}$ Similarly, 677CT polymorphism is associated with risk of myocardial infarction in African, North American and elderly populations. ${ }^{12}$

According to Cortese and Motti (2001), homozygosity for the Val allele there appears to be a relative deficiency in the re-methylation process of homocysteine which lead to a 
mild to moderate hyper-homocysteinaemia. This condition can be a possible risk factor for atherosclerosis. The genetic influence of the MTHFR polymorphism on homocysteine levels is reduced in females in premenopausal age and persons with higher serum levels of folate or vitamin B12. The independent role of MTHFR polymorphism in cardiovascular risk is still not well established.

Mutations in the MTHFR gene could be one of the factors leading to increased risk of developing schizophrenia. ${ }^{13}$ Similarly, there is also reported link between MTHFR mutations and dementia ${ }^{14}$ and Alzheimer's disease in Asians. ${ }^{15}$

\section{A1298C SNP (Glu ${ }^{429}$ Ala)}

There are two possibilities either $\mathrm{A}$ or $\mathrm{C}$ at nucleotide 1298 of the MTHFR. 1298A (leading to a Glu at amino acid 429 ) is the most common while 1298C (leading to an Ala substitution at amino acid 429) is less common. $1298 \mathrm{AA}$ is the 'normal' homozygous, 1298 AC the 'heterozygous' and $1298 \mathrm{CC}$ the homozygous for the 'variant'. The C mutation does not affect the MTHFR protein, homocysteine levels and does not result in thermolabile MTHFR. It, however, affects the conversion of MTHF to tetrahydrobiopterin, an important cofactor in the production of neurotransmitters and the synthesis of nitric oxide.

A maternal MTHFR 1298AC polymorphism is associated with Down Syndrome pregnancy particularly in Asian population. ${ }^{5}$ 1298AC polymorphism may also play a role in the development of major depressive disorder. ${ }^{16}$

Hyper-homocysteinemia is also associated with high blood pressure, glaucoma, and ischaemic stroke. ${ }^{17}$ This condition is also linked to migraines and mental disorders like bipolar disorder, and depression is linked to inadequate methylation resulting from MTHFR polymorphism. ${ }^{18,19}$

Severe MTHFR deficiency is rare but can be caused by mutations resulting in $0-20 \%$ residual enzyme activity. ${ }^{6}$ The affected persons exhibit developmental delay, motor and gait dysfunction, seizures and neurological impairment and have extremely high levels of plasma and urine homocysteine and low plasma methionine levels.

\section{Biochemical impact of MTHFR Deficiency}

1. Hyper-homocysteinemia: The mutated genotype is linked to low serum folate levels and hyperhomocysteinemia and homocysteinuria. This is associated with toxic effects on vasculature leading to thromboembolic phenomenon, premature atherosclerosis and coronary artery disease..$^{8,20}$

2. Dysregulation in methionine and S-adenosylmethionine formation: Reduced or deficient activity of MTHFR enzyme disrupts $\mathrm{S}$-adenosylmethionine synthesis rather conversion of homocysteine to Sadenosylhomocysteine might interfere with many other methyltransferases. Transfer of methyl groups are an important aspect in DNA and histone methylation processes, involved in the process of epigenesis and imprinting. Alteration in these processes could be one of the major influencing factors for neural tube defects, increased chromosomal anomalies, early fetal loss or habitual first trimester abortions..$^{21,22}$

3. Alteration in folate metabolism: Low methytetrahydrofolate levels and an increase in other forms of folate like methylenetetrahydrofolate and nonmethylated and formylated forms, have been associated with redistribution of active folates. This might limit the bioavailability of purines and pyrimidines required for DNA synthesis and repair during cell cycle. This could be a potential factor for oncogenesis, psoriasis and developmental delay in children. This could also be a contributory factor for neurological and psychiatric diseases associated with MTHR diseases. ${ }^{21-24}$

4. Accumulation of unmetabolised folic acid: Accumulation of other forms of folate and unmetabilosed folic acid (UMFA) in red blood cells (RBC) and lymphocytes have been associated with altered immunity and inflammatory diseases. Folic acid supplementation in MTHFR deficient individuals could significantly elevate UMFA levels having deleterious effect on natural killer (NK) cells decreasing natural immunity. ${ }^{24}$

\section{Clinical Impact of MTHFR Deficiency}

MTHFR polymorphism has been associated with various diseases as given below:

1. Vascular disease: Hyper-homocysteinemia has been marked as an independent factor for coronary artery disease (CAD) and ischemic stroke. Few studies have reported regarding hemorrhagic stroke, essential hypertension, retinal vein occlusion and venous thromboembolism. ${ }^{8,25}$ The low serum folate levels and hyper-homocyteinemia and homocysteinuria can be linked to this mutated genotype. Toxic effects on the vasculature lead to thromboembolic phenomenon and coronary artery disease.

2. Infertility and reproduction: A strong impact of MTHFR polymorphism has been reported in patients with habitual abortions. Hyper-homocysteinemia leading to hypercoaguable state could be the most possible cause for the first trimester fetal loss. Repeated Assisted reproductive (ART) failures have been observed. MTHFR isoforms also have an impact on the sperm quality and quantity and also on low ovarian reserve. ${ }^{21,24}$ First trimester fetal abortions are the results of alterations in the process of epigenesis and imprinting related to DNA and histone methylation process (reduced or deficient activity of MTHFR enzyme).

3. Neural tube defects: Chromosomal abnormalities and neural tube defects have been well recognised with folate deficiencies and as well as MTHFR polymorphisms. ${ }^{11,20}$ Reduced activity of MTHFR enzyme disrupts S-adenosylmethionine synthesis which in turn might interfere with many other methyltransferases. Significant changes in these biochemical processes could be an etiological factor for neural tube defects. 
4. Neurological and psychiatric diseases: Neurotoxic effects of hyper-homocysteinemia leading to hyperexcitibility have been related to Parkinsonism and Alzheimer's diseases. Risk of schizophrenia and bipolar disorders are also associated with MTHFR polymorphism. ${ }^{13,19}$ Decreased methytetrahydrofolate levels accompanied by increased methylenetetrahydrofolate and non-methylated and formylated forms, affect redistribution of active folates thus limiting the bioavailability of purines and pyrimidines required for DNA synthesis and repair during cell cycle. Neurological and psychiatric diseases could be a result of this process involving MTHR diseases.

5. Inflammatory diseases: Elevated homocysteine and accumulation of non-methylated and formyl tetrahydrofolates in neuronal tissues have been reported in individuals with MTHFR polymorphism and multiple sclerosis or sporadic amyotropic lateral sclerosis. High levels of serum inflammatory markers like IL-1 $\beta, \mathrm{TNF} \alpha$ and CRF were reported in these patients. ${ }^{26}$

6. Cancer: Altered methylation processes required for DNA synthesis and repair could be the possible mechanism for oncogenesis. Psoriasis, altered epithelial repair have been seen in MTHFR polymorphism individuals. ${ }^{910}$ Studies have revealed that low methytetrahydrofolate levels and an increase in other forms of folate limit the bioavailability of purines and pyrimidines required for DNA synthesis and repair during cell cycle. This disturbed biochemical condition could lead to oncogenesis.

\section{Conclusion}

MTHFR enzyme is a key enzyme for folate metabolism. Numerous polymorphisms have been reported for the gene coding for the enzyme. Reduced or deficient activity of the enzyme results in hyperhomocysteinemia. MTHFR polymorphism has been associated with various diseases like vascular disease, infertility, reproductive failure, neurological diseases, cancers and inflammatory diseases. Folate supplementation in MTHFR deficient individuals might result in accumulation of UMFA leading to immune deficiency. Supplementation of active folate, 5MTHF could be a better alternative as compared to folic acid supplementation.

\section{Conflict of Interest: None.}

\section{References}

1. Shahzad K, Hai A, Ahmed A, Kizilbash N, Aruwaili J. A Structured-based Model for the Decreased Activity of Ala222Val and Glu429Ala Methylenetetrahydrofolate Reductase (MTHFR) Mutants. Bioinformation 2013;9(18):929-936.

2. Froese DS, Kopec J, Rembeza E, Bezerra GA, Oberholzer AE, Suormala T, et al. Structural basis for the regulation of human 5,10 -methylenetetrahydrofolate reductase by phosphorylation and S-adenosylmethionine inhibition. Nat Commun 2018;9(1):2261.

3. Bellamy MF, McDowell IF. Putative mechanisms for vascular damage by homocysteine. J Inherit Metab Dis 1997;20(2):307-315.

4. Reilly R, McNulty H, Pentieva K, Strain JJ, Ward M. MTHFR 677TT genotype and disease risk: is there a modulating role for B-vitamins? Proc Nutr Soc 2014;73(1):47-56.

5. Rai V, Yadav U, Kumar P. Null association of maternal MTHFR A1298C polymorphism with Down syndrome pregnancy: An updated meta-analysis. Egypt J Med Hum Genet 2017;18(1):9-18.

6. Sibani S, Christensen B, O’Ferrall E, Saadi I, Hiou-Tim F, Rosenblatt DS, et al. Characterization of six novel mutations in the methylenetetrahydrofolate reductase (MTHFR) gene in patients with homocystinuria. Hum Mutat 2000;15(3):280287.

7. Schneider JA, Rees DC, Liu YT, Clegg JB. Worldwide distribution of a common methylenetetrahydrofolate reductase mutation. Am J Hum Genet 1998;62(5):1258-1260.

8. Frosst P, Blom HJ, Milos R, Goyette P, Sheppard CA, Matthews RG, et al. A candidate genetic risk factor for vascular disease: a common mutation in methylenetetrahydrofolate reductase. Nat Genet 1995;10(1):111-113.

9. Ojha RP, Gurney JG. Methylenetetrahydrofolate reductase C677T and overall survival in pediatric acute lymphoblastic leukemia: a systematic review. Leuk Lymphoma 2014;55(1):67-73.

10. Bailey LB. Folate, methyl-related nutrients, alcohol, and the MTHFR 677C--> T polymorphism affect cancer risk: intake recommendations. J Nutr 2003;133(11 Suppl 1):3748S-3753S.

11. Wu X, Zhao L, Zhu H, He D, Tang W, Luo Y. Association between the MTHFR C677T polymorphism and recurrent pregnancy loss: a meta-analysis. Genet Test Mol Biomark 2012;16(7):806-11.

12. Alizadeh S, Djafarian K, Moradi S, Shab-Bidar S. C667T and A1298C polymorphisms of methylenetetrahydrofolate reductase gene and susceptibility to myocardial infarction: A systematic review and meta-analysis. Int J Cardiol 2016;217:99-108.

13. Zhang Y, Yan H, Tian L, Wang F, Lu T, Wang L, et al. Association of MTHFR C677T polymorphism with schizophrenia and its effect on episodic memory and gray matter density in patients. Behav Brain Res 2013;243:146-152.

14. Nishiyama M, Kato Y, Hashimoto M, Yukawa S, Omori K. Apolipoprotein E, methylenetetrahydrofolate reductase (MTHFR) mutation and the risk of senile dementia--an epidemiological study using the polymerase chain reaction (PCR) method. J Epidemiol 2000;10(3):163-172.

15. Hua Y, Zhao H, Kong Y, Ye M. Association between the MTHFR gene and Alzheimer's disease: a meta-analysis. Int $J$ Neurosci 2011;121(8):462-471.

16. Cho K, Amin ZM, An J, Rambaran KA, Johnson TB, Alzghari SK. Methylenetetrahydrofolate Reductase A1298C Polymorphism and Major Depressive Disorder. Cureus 2017;9(10):e1734.

17. Li P, Qin C. Methylenetetrahydrofolate reductase (MTHFR) gene polymorphisms and susceptibility to ischemic stroke: a meta-analysis. Gene 2014;535(2):359-364.

18. Azimova JE, Sergeev AV, Korobeynikova LA, Kondratieva NS, Kokaeva ZG, Shaikhaev GO, et al. Effects of MTHFR gene polymorphism on the clinical and electrophysiological characteristics of migraine. BMC Neurol 2013;13:103.

19. Gilbody S, Lewis S, Lightfoot T. Methylenetetrahydrofolate reductase (MTHFR) genetic polymorphisms and psychiatric disorders: a HuGE review. Am J Epidemiol 2007;165(1):1-13. 
20. Matthews RG, Sheppard C, Goulding C.

Methylenetetrahydrofolate reductase and methionine synthase: biochemistry and molecular biology. Eur J Pediatr 1998;157(S2):S54-59.

21. Liew S-C, Gupta ED. Methylenetetrahydrofolate reductase (MTHFR) C677T polymorphism: Epidemiology, metabolism and the associated diseases. Eur J Med Genet 2015;58(1):1-10.

22. Leclerc D, Sibani S, Rozen R. Molecular Biology of Methylenetetrahydrofolate Reductase (MTHFR) and Overview of Mutations/Polymorphisms [Internet]. Landes Bioscience; 2013 [cited 2019 Jan 26]. Available from: https://www.ncbi.nlm.nih.gov/books/NBK6561/

23. MTHFR - Methylenetetrahydrofolate reductase - Homo sapiens (Human) - MTHFR gene \& protein [Internet]. [cited 2019 Jan 26]. Available from: https://www.uniprot.org/uniprot/Q5SNW5
24. Servy E, Menezo Y. The Methylene Tetrahydrofolate Reductase (MTHFR) isoform challenge. High doses of folic acid are not a suitable option compared to 5 Methyltetrahydrofolate treatment. Clin Obstet Gynecol Reprod Med 2017;3(6).

25. Selzer RR, Rosenblatt DS, Laxova R, Hogan K. Adverse Effect of Nitrous Oxide in a Child with 5,10Methylenetetrahydrofolate Reductase Deficiency. N Engl J Med 2003;349(1):45-50.

26. Alatab S, Hossein-nezhad A, Mirzaei K, Mokhtari F, Shariati G, Najmafshar A. Inflammatory profile, age of onset, and the MTHFR polymorphism in patients with multiple sclerosis. $J$ Mol Neurosci MN 2011;44(1):6-11. 